\title{
Elevated serum magnesium associated with SGLT2 inhibitor use in type 2 diabetes patients: a meta-analysis of randomised controlled trials
}

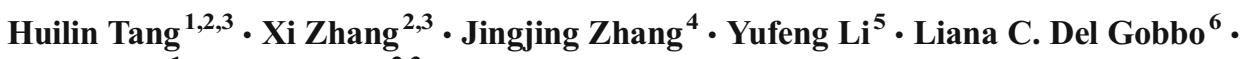 \\ Suodi Zhai ${ }^{1} \cdot$ Yiqing Song ${ }^{2,3}$
}

Received: 10 June 2016 / Accepted: 18 August 2016/Published online: 15 September 2016

(C) Springer-Verlag Berlin Heidelberg 2016

\begin{abstract}
Aims/hypothesis By analysing available evidence from randomised controlled trials (RCTs), we aimed to examine whether and to what extent sodium-glucose cotransporter 2 (SGLT2) inhibitors affect serum electrolyte levels in type 2 diabetes patients.

Methods We searched PubMed, EMBASE, Cochrane Central Register of Controlled Trials (CENTRAL) and ClinicalTrials.gov up to 24 May 2016 for published RCTs of SGLT2 inhibitors that reported changes in serum electrolyte levels. Weighted mean differences (WMD) between each SGLT2 inhibitor and placebo were calculated using a randomeffects model. Dose-dependent relationships for each SGLT2 inhibitor were evaluated using meta-regression analysis.
\end{abstract}

Electronic supplementary material The online version of this article (doi:10.1007/s00125-016-4101-6) contains peer-reviewed but unedited supplementary material, which is available to authorised users.

Yiqing Song

yiqsong@iu.edu

1 Department of Pharmacy, Peking University Third Hospital, Beijing, People's Republic of China

2 Department of Epidemiology, Richard M. Fairbanks School of Public Health, Indiana University, 1050 Wishard Blvd,

Indianapolis, IN 46202, USA

3 Center for Pharmacoepidemiology, Richard M. Fairbanks School of Public Health, Indiana University, Indianapolis, IN, USA

4 Division of Nephrology, Sidney Kimmel Medical College, Thomas Jefferson University, Philadelphia, PA, USA

5 Department of Endocrinology, Beijing Pinggu Hospital, Beijing, People's Republic of China

6 Department of Medicine, Division of Cardiovascular Medicine, Stanford University, Stanford, CA, USA
Results Eighteen eligible RCTs, including 15,309 patients and four SGLT2 inhibitors (canagliflozin, dapagliflozin, empagliflozin and ipragliflozin) were evaluated. In patients without chronic kidney disease, each SGLT2 inhibitor significantly increased serum magnesium levels compared with placebo (canagliflozin: WMD $0.06 \mathrm{mmol} / 1$ for $100 \mathrm{mg}$ and $0.09 \mathrm{mmol} / 1$ for $300 \mathrm{mg}$; dapagliflozin: WMD $0.1 \mathrm{mmol} / 1$ for $10 \mathrm{mg}$; empagliflozin: WMD $0.04 \mathrm{mmol} / 1$ for $10 \mathrm{mg}$ and $0.07 \mathrm{mmol} / 1$ for $25 \mathrm{mg}$; and ipragliflozin: WMD $0.05 \mathrm{mmol} / \mathrm{l}$ for $50 \mathrm{mg}$ ). Canagliflozin increased serum magnesium in a linear dose-dependent manner $(p=0.10)$. Serum phosphate was significantly increased by dapagliflozin. Serum sodium appeared to significantly differ by SGLT2 inhibitor type. No significant changes in serum calcium and potassium were observed. Findings were robust after including trials involving patients with chronic kidney disease.

Conclusions/interpretation SGLT2 inhibitors marginally increased serum magnesium levels in type 2 diabetes patients indicating a drug class effect. Further investigations are required to examine the clinical significance of elevated magnesium levels in individuals with type 2 diabetes.

Keywords Meta-analysis $\cdot$ Serum electrolytes $\cdot$ Serum magnesium $\cdot$ SGLT2 inhibitors · Type 2 diabetes
Abbreviations
CKD Chronic kidney disease
RCTs Randomised controlled trials
SGLT2 Sodium-glucose cotransporter 2
UGE Urinary glucose excretion
WMD Weighted mean difference 


\section{Introduction}

Electrolyte abnormalities, usually as a result of renal impairment, can lead to serious complications and even death. Sodium-glucose cotransporter 2 (SGLT2) inhibitors are a novel class of glucose-lowering agents that are indicated for the treatment of type 2 diabetes. SGLT2 inhibitors selectively inhibit renal glucose reabsorption and increase urinary glucose excretion (UGE) [1]. As a consequence of increased UGE, treatment with SGLT2 inhibitors may result in osmotic diuresis, which may trigger volume depletion and dehydration [2]. However, data on the influence of such haemodynamic changes on renal electrolyte handling in individuals with type 2 diabetes is lacking. We conducted a meta-analysis of the available evidence from randomised controlled trials (RCTs) to examine whether and to what extent SGLT2 inhibitors affect serum electrolyte levels in patients with type 2 diabetes.

\section{Methods}

Search strategy and selection of articles We searched the PubMed, EMBASE, Cochrane Central Register of Controlled Trials (CENTRAL) and ClinicalTrials.gov databases up to 24 May 2016 to identify eligible RCTs using relevant search terms without restrictions on language and year of publication (see ESM Table 1 for a complete list of search terms). We included parallel-design RCTs of at least 24 weeks duration that compared SGLT2 inhibitors to placebo in adults with type 2 diabetes and reported mean (percentage) change from baseline in electrolyte levels for each group or data that enabled calculation of these variables. Our primary outcome was mean (percentage) change from baseline in serum magnesium level, and our secondary outcomes included mean (percentage) change from baseline in serum sodium, phosphate, potassium and calcium levels.

Data extraction and quality assessment We collected the following information from each eligible RCT: first author and publication year, study characteristics (country of origin, design and funding), patient characteristics (inclusion criteria, background treatments, mean age, pre-existing chronic kidney disease [CKD], race, baseline $\mathrm{HbA}_{1 \mathrm{c}}$, mean eGFR and BMI), interventions (SGLT2 inhibitor type and dose), mean electrolyte levels, variance measure and the number of participants in the treatment and placebo arms for all reported periods. Mean (SD) change from baseline in $\mathrm{mmol} / \mathrm{l}$ was extracted for each SGLT2 inhibitor, with the exception of canagliflozin, for which data were presented as mean (SD) percentage change from baseline in the original article. The Cochrane risk of bias tool was used to assess the quality of the RCTs based on five domains: random sequence generation (selection bias), allocation concealment (selection bias), blinding (performance bias and detection bias), incomplete outcome data (attrition bias) and selective reporting (reporting bias). Two reviewers ( $\mathrm{H}$. Tang and X. Zhang) independently extracted the data and assessed the quality of each RCT. Any disagreements were resolved by consensus or referral to a third reviewer (Y. Song).

Statistical analysis Weighted mean differences (WMD) (95\% CI) in serum electrolyte levels were calculated for each SGLT2 inhibitor and placebo using a random-effects model to evaluate each SGLT2 inhibitor separately and by dose. Heterogeneity was quantified using $I^{2}$, with an $I^{2}$ of 25,50 and 75 indicating low, medium and high heterogeneity, respectively. A meta-regression analysis was employed to investigate any possible dose-dependent relationships between each SGLT2 inhibitor and changes in serum electrolyte levels [3]. The main meta-analysis was performed in patients without CKD. An additional sensitivity analysis was performed to include the RCTs that involved individuals with CKD. Publication bias was assessed using Begg's test. All statistical analyses were performed with STATA (version 14; StataCorp, College Station, TX, USA).

\section{Results}

Of 1874 articles screened, 18 trials met the eligibility criteria (ESM Fig. 1), totalling 15,309 unique participants [4-21]. Four SGLT2 inhibitors (canagliflozin, dapagliflozin, empagliflozin and ipragliflozin) were evaluated. The duration of interventions ranged from 24 to 260 weeks (mean duration: 70 weeks). Participants were generally middle-aged (mean age: 58 ), white (14/18 studies) and without CKD (16/18 studies) (ESM Table 2). Baseline electrolyte levels were within normal reference ranges for all studies (ESM Table 3). Most of the trials included in the meta-analysis were judged to have a low risk of bias, except for one trial that had a high risk of bias due to the lack of blinding [10] (ESM Fig. 2).

For patients receiving canagliflozin, serum magnesium levels were significantly increased compared with those receiving placebo (100 mg: WMD $8.03 \%$ [95\% CI 6.89, 9.16]; $300 \mathrm{mg}$ : WMD $11.06 \%$ [95\% CI 9.92, 12.19]) (Fig. 1a). Canagliflozin increased serum magnesium in a dose-dependent manner (for linear trend $p=0.10$ ). Serum magnesium levels were also significantly increased relative to placebo in participants receiving empagliflozin $(10 \mathrm{mg}$ : WMD $0.04 \mathrm{mmol} / 1$ [95\% CI 0.02, 0.07]; $25 \mathrm{mg}$ : WMD $0.07 \mathrm{mmol} / 1[95 \% \mathrm{CI} 0.04,0.09])$ and ipragliflozin $(50 \mathrm{mg}$ : WMD $0.05 \mathrm{mmol} / 1$ [95\% CI $0.03,0.08])$. Of the participants receiving dapagliflozin, only those in the $10 \mathrm{mg}$ group had significantly increased serum magnesium levels relative to placebo (WMD $0.10 \mathrm{mmol} / 1$ [95\% CI 0.01, 0.19]). 
Fig. 1 Meta-analysis of WMD ( $95 \% \mathrm{CI}$ ) for changes in serum magnesium level for SGLT2 inhibitors vs placebo, stratified by dose. (a) WMD (95\% CI) for canagliflozin calculated as mean percentage change from baseline. (b) WMD (95\% CI) for dapagliflozin, empagliflozin and ipragliflozin calculated as mean change from baseline in $\mathrm{mmol} / \mathrm{l}$. Rosenstock et al (2015) was excluded from the meta-analysis because of an SD of 0 . WMD are from a random-effects model analysis a

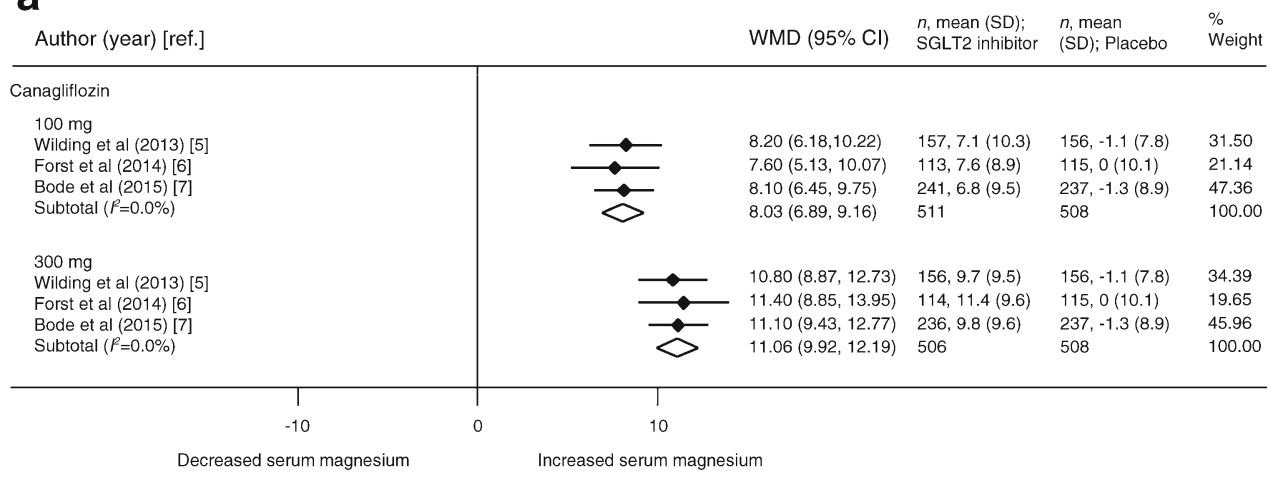

b

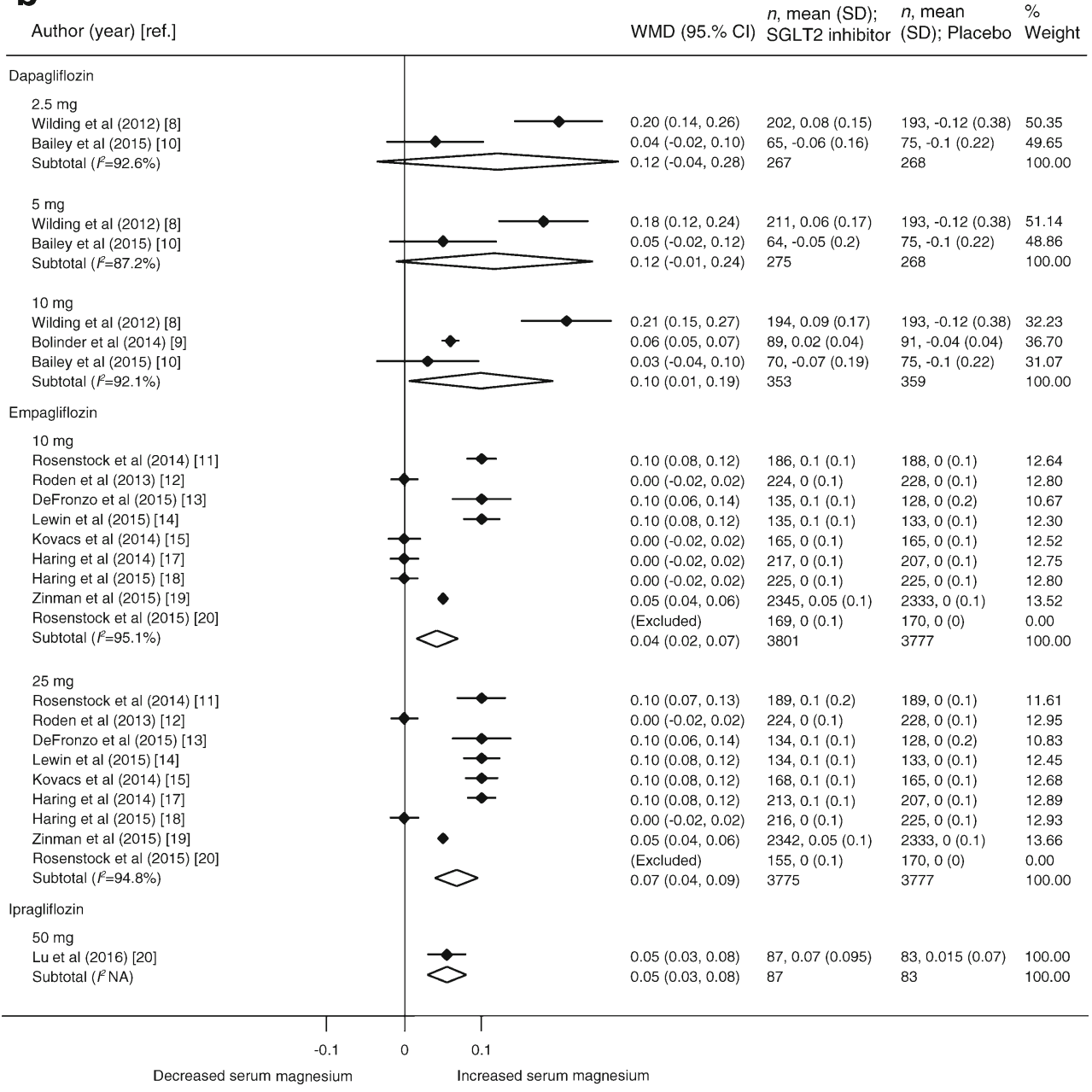

An additional sensitivity analysis that included two trials that involved patients with CKD showed that the results of the analysis were robust (data not shown).

Statistically significant between-study heterogeneity was evident for the dapagliflozin and empagliflozin RCTs $\left(I^{2}>75 \%\right)$, but not for the canagliflozin RCTs $\left(I^{2}=0 \%\right)$. There was no evidence of publication bias for serum magnesium in this meta-analysis $(p>0.05)$.
Serum phosphate levels were significantly increased compared with placebo for dapagliflozin $5 \mathrm{mg}$ (WMD $0.04 \mathrm{mmol} / \mathrm{l}$ [95\% CI 0.01, 0.06]) and $10 \mathrm{mg}$ (WMD $0.05 \mathrm{mmol} / \mathrm{l}[95 \% \mathrm{CI}$ 0.02, 0.09]) (Table 1 and ESM Fig. 3). Serum sodium levels were significantly higher with empagliflozin $25 \mathrm{mg}$ relative to placebo (WMD $0.31 \mathrm{mmol} / 1$ [95\% CI 0.04, 0.58]). However, serum sodium levels were reduced with canagliflozin $300 \mathrm{mg}$ (WMD $-0.36 \mathrm{mmol} / 1[95 \% \mathrm{CI}-0.68,-0.05]$ ) (Table 1 and ESM 


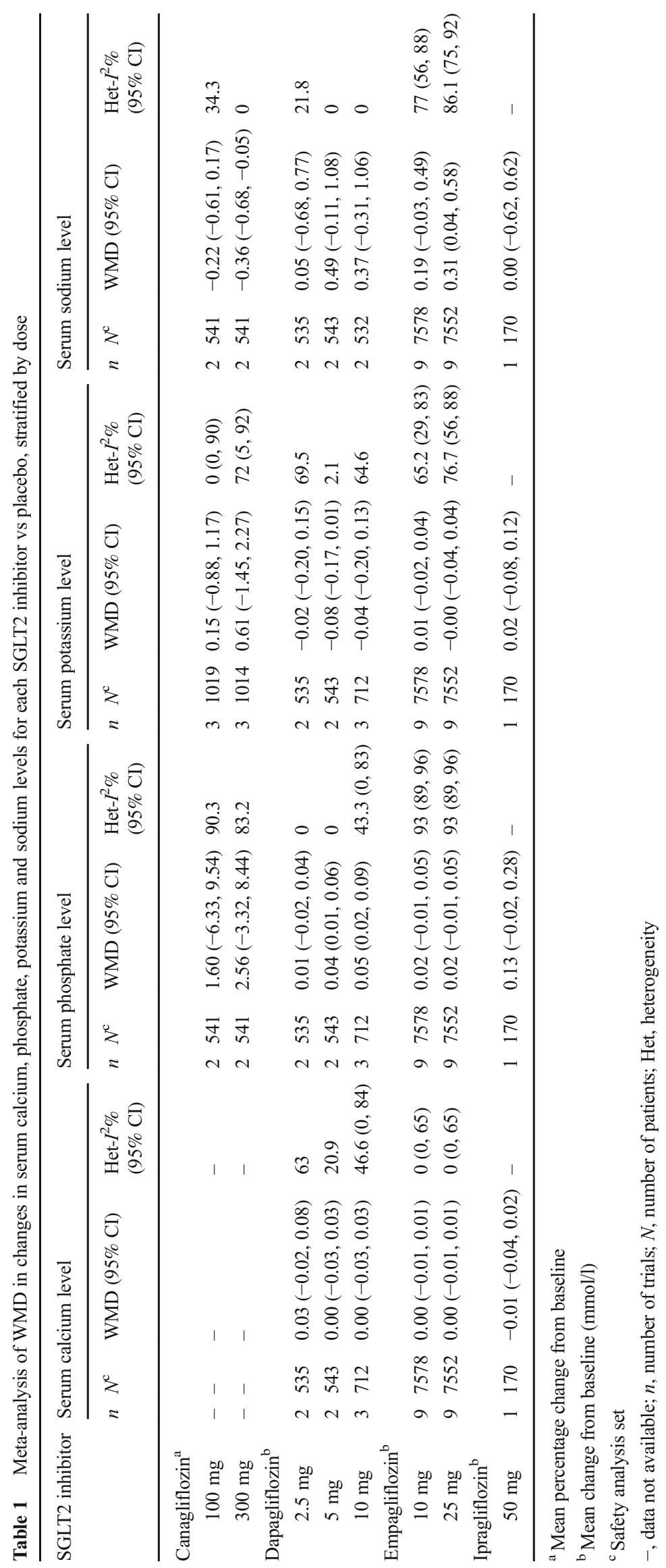


Fig. 4). No significant changes in serum calcium and potassium levels were observed among the participants receiving SGLT2 inhibitors (Table 1, ESM Figs 5, 6). A dose-dependent relationship between the SGLT2 inhibitors and electrolyte levels was not observed. When the trials involving participants with CKD were included in the analysis, all of the results were similar (data not shown), with one exception. The mean change from baseline in serum sodium level with empagliflozin $10 \mathrm{mg}$ was significantly different from placebo (WMD $0.26 \mathrm{mmol} / \mathrm{l}$ [95\% CI 0.03, 0.49]). Heterogeneity was variable across SGLT2 type, dose and electrolyte level (Table 1).

\section{Discussion}

Our meta-analysis of $18 \mathrm{RCTs}$ involving 15,309 patients provides the first robust evidence that SGLT2 inhibitors significantly increase serum magnesium levels in patients with type 2 diabetes. On average, serum magnesium levels were raised by $0.06 \mathrm{mmol} / 1$ for canagliflozin $100 \mathrm{mg}, 0.09 \mathrm{mmol} / \mathrm{l}$ for canagliflozin $300 \mathrm{mg}, 0.1 \mathrm{mmol} / \mathrm{l}$ for dapagliflozin $10 \mathrm{mg}$, $0.04 \mathrm{mmol} / 1$ for empagliflozin $10 \mathrm{mg}, 0.07 \mathrm{mmol} / 1$ for empagliflozin $25 \mathrm{mg}$ and $0.05 \mathrm{mmol} / 1$ for ipragliflozin $50 \mathrm{mg}$. Taken together, these results indicate that there is a drug class effect on serum magnesium levels. Furthermore, effects on serum phosphate and sodium levels differ by SGLT2 inhibitor type and dose. However, no significant effects on serum calcium and potassium levels were observed.

Given that SGLT2 inhibitors induce glucosuria and osmotic diuresis, which may trigger volume depletion and dehydration, we hypothesised that changes in circulating electrolyte levels might occur with the use of such medications. The significant elevations in serum magnesium levels, and the possibility of increased phosphate levels, might be the result of osmotic diuresis caused by SGLT2 inhibitors, but the precise mechanisms involved are unknown. Abnormally high magnesium levels are predictive of total mortality in individuals with heart failure [22], those who are critically ill [23] and those receiving haemodialysis [24]. Therefore, caution must be exercised in patients with impaired renal function, such as in severe CKD. On the other hand, both in the general population and in people with type 2 diabetes, a gradient of risk for cardiovascular disease has been observed across the normal range of serum magnesium [25], with concentrations at the higher end of the normal range associated with a lower risk of cardiovascular events [25]. Our meta-analysis found that a mean increase of $0.05 \mathrm{mmol} / \mathrm{l}$ in serum magnesium was significantly associated with a reduction in systolic BP by $2.00 \mathrm{mmHg}$ and diastolic BP by $1.78 \mathrm{mmHg}$ compared with placebo [26]. If serum magnesium is causally related to cardiovascular risk, a modest increase in serum magnesium could have contributed to a reduction in cardiovascular mortality observed among participants with type 2 diabetes in the EMPA-REG OUTCOME trial
[19]. However, the observed changes in serum magnesium levels were, on average, within the physiological range. We do not know what proportion of individuals have serum magnesium levels above the normal range, and therefore, the clinical significance/interpretation of these data is uncertain.

Changes in serum phosphate were also observed for dapagliflozin with a mean increase of $0.04 \mathrm{mmol} / \mathrm{l}$ and $0.05 \mathrm{mmol} / 1$ for $5 \mathrm{mg}$ and $10 \mathrm{mg}$, respectively. Increased serum phosphate may have adverse effects on bone health by increasing secretion of the parathyroid hormone, which enhances bone resorption and increases the risk of bone fractures [27]. Although one study demonstrated that canagliflozin was associated with a decrease in bone mineral density at total hip in individuals with type 2 diabetes [28], the effects of SGLT2 inhibitors on bone health are still uncertain. It is interesting to find that serum sodium levels appear to differ by SGLT2 inhibitor type with a mean reduction of $0.36 \mathrm{mmol} / \mathrm{l}$ for canagliflozin $300 \mathrm{mg}$ and a mean increase of $0.31 \mathrm{mmol} / \mathrm{l}$ for empagliflozin $25 \mathrm{mg}$. However, the clinical significance of a small change in serum sodium level $<0.5 \mathrm{mmol} / \mathrm{l}$ is still unclear.

Some limitations of our meta-analysis, which may reduce the strength of the evidence, merit discussion (e.g. the low number of participants included for each SGLT2 inhibitor and statistically significant between-study heterogeneity). Furthermore, we were unable to calculate the proportion of individuals who have abnormal electrolyte levels as a result of receiving SGLT2 inhibitor treatment because of limited information in the trial reports.

In summary, SGLT2 inhibitors marginally increase serum magnesium levels in individuals with type 2 diabetes. Dapagliflozin also increased serum phosphate levels, and serum sodium levels appeared to differ between empagliflozin and canagliflozin. Further investigations are required to examine the clinical significance of changes in serum magnesium, phosphate and sodium caused by SGLT2 inhibitors in patients with type 2 diabetes, especially in individuals with chronic comorbid diseases.

Funding The project described was supported by the Indiana University Health-Indiana University School of Medicine Strategic Research Initiative.

Duality of interest The authors declare that there is no duality of interest associated with this manuscript.

Contribution statement HT and YS designed the study. HT and XZ identified and acquired reports of trials and extracted the data. HT and XZ performed all data analyses, checked for statistical inconsistency and interpreted the data. HT, XZ, JZ, YL, LDG, SZ and YS contributed to data interpretation. HT drafted the report and all other authors (XZ, JZ, YL, SZ, LDG and YS) critically reviewed the report. HT, XZ, JZ, YL, LDG, SZ and YS approved the final manuscript. YS is responsible for the integrity of the work as a whole. 


\section{References}

1. Ferrannini E, Solini A (2012) SGLT2 inhibition in diabetes mellitus: rationale and clinical prospects. Nat Rev Endocrinol 8: 495-502

2. Weir MR, Kline I, Xie J, Edwards R, Usiskin K (2014) Effect of canagliflozin on serum electrolytes in patients with type 2 diabetes in relation to estimated glomerular filtration rate (eGFR). Curr Med Res Opin 30:1759-1768

3. Harbord RM, Higgins JPT (2008) Meta-regression in Stata. Stata J 8:493-519

4. Yale JF, Bakris G, Cariou B et al (2014) Efficacy and safety of canagliflozin over 52 weeks in patients with type 2 diabetes mellitus and chronic kidney disease. Diabetes Obes Metab 16:1016-1027

5. Wilding JP, Charpentier G, Hollander P et al (2013) Efficacy and safety of canagliflozin in patients with type 2 diabetes mellitus inadequately controlled with metformin and sulphonylurea: a randomised trial. Int J Clin Pract 67:1267-1282

6. Forst T, Guthrie R, Goldenberg R et al (2014) Efficacy and safety of canagliflozin over 52 weeks in patients with type 2 diabetes on background metformin and pioglitazone. Diabetes Obes Metab 16:467-477

7. Bode B, Stenlof K, Harris S et al (2015) Long-term efficacy and safety of canagliflozin over 104 weeks in patients aged 55-80 years with type 2 diabetes. Diabetes Obes Metab 17:294-303

8. Wilding JP, Woo V, Soler NG et al (2012) Long-term efficacy of dapagliflozin in patients with type 2 diabetes mellitus receiving high doses of insulin: a randomized trial. Ann Intern Med 156: 405-415

9. Bolinder J, Ljunggren O, Johansson L et al (2014) Dapagliflozin maintains glycaemic control while reducing weight and body fat mass over 2 years in patients with type 2 diabetes mellitus inadequately controlled on metformin. Diabetes Obes Metab 16:159-169

10. Bailey CJ, Morales Villegas EC, Woo V, Tang W, Ptaszynska A, List JF (2015) Efficacy and safety of dapagliflozin monotherapy in people with type 2 diabetes: a randomized double-blind placebocontrolled 102-week trial. Diabet Med 32:531-541

11. Rosenstock J, Jelaska A, Frappin G et al (2014) Improved glucose control with weight loss, lower insulin doses, and no increased hypoglycemia with empagliflozin added to titrated multiple daily injections of insulin in obese inadequately controlled type 2 diabetes. Diabetes Care 37:1815-1823

12. Roden M, Weng J, Eilbracht J et al (2013) Empagliflozin monotherapy with sitagliptin as an active comparator in patients with type 2 diabetes: a randomised, double-blind, placebo-controlled, phase 3 trial. Lancet Diabetes Endocrinol 1:208-219

13. DeFronzo RA, Lewin A, Patel S et al (2015) Combination of empagliflozin and linagliptin as second-line therapy in subjects with type 2 diabetes inadequately controlled on metformin. Diabetes Care 38:384-393

14. Lewin A, DeFronzo RA, Patel S et al (2015) Initial combination of empagliflozin and linagliptin in subjects with type 2 diabetes. Diabetes Care 38:394-402
15. Kovacs CS, Seshiah V, Swallow R et al (2014) Empagliflozin improves glycaemic and weight control as add-on therapy to pioglitazone or pioglitazone plus metformin in patients with type 2 diabetes: a 24-week, randomized, placebo-controlled trial. Diabetes Obes Metab 16:147-158

16. Barnett AH, Mithal A, Manassie J et al (2014) Efficacy and safety of empagliflozin added to existing antidiabetes treatment in patients with type 2 diabetes and chronic kidney disease: a randomised, double-blind, placebo-controlled trial. Lancet Diabetes Endocrinol 2:369-384

17. Haring HU, Merker L, Seewaldt-Becker E et al (2014) Empagliflozin as add-on to metformin in patients with type 2 diabetes: a 24-week, randomized, double-blind, placebo-controlled trial. Diabetes Care 37:1650-1659

18. Haring HU, Merker L, Christiansen AV et al (2015) Empagliflozin as add-on to metformin plus sulphonylurea in patients with type 2 diabetes. Diabetes Res Clin Pract 110:82-90

19. Zinman B, Wanner C, Lachin JM et al (2015) Empagliflozin, cardiovascular outcomes, and mortality in type 2 diabetes. $\mathrm{N}$ Engl J Med 373:2117-2128

20. Rosenstock J, Jelaska A, Zeller C, Kim G, Broedl UC, Woerle HJ (2015) Impact of empagliflozin added on to basal insulin in type 2 diabetes inadequately controlled on basal insulin: a 78-week randomized, double-blind, placebo-controlled trial. Diabetes Obes Metab 17:936-948

21. Lu CH, Min KW, Chuang LM, Kokubo S, Yoshida S, Cha BS (2016) Efficacy, safety, and tolerability of ipragliflozin in Asian patients with type 2 diabetes mellitus and inadequate glycemic control with metformin: results of a phase 3 randomized, placebo-controlled, double-blind, multicenter trial. J Diabetes Investig 7:366373

22. Corbi G, Acanfora D, Iannuzzi GL et al (2008) Hypermagnesemia predicts mortality in elderly with congestive heart disease: relationship with laxative and antacid use. Rejuvenation Res 11:129-138

23. Haider DG, Lindner G, Ahmad SS et al (2015) Hypermagnesemia is a strong independent risk factor for mortality in critically ill patients: results from a cross-sectional study. Eur J Intern Med 26: 504-507

24. Lacson E Jr, Wang W, Ma L, Passlick-Deetjen J (2015) Serum magnesium and mortality in hemodialysis patients in the United States: a cohort study. Am J Kidney Dis 66:1056-1066

25. Del Gobbo LC, Imamura F, Wu JH, de Oliveira Otto MC, Chiuve SE, Mozaffarian D (2013) Circulating and dietary magnesium and risk of cardiovascular disease: a systematic review and metaanalysis of prospective studies. Am J Clin Nutr 98:160-173

26. Zhang X, Li Y, Del Gobbo LC et al (2016) Effects of magnesium supplementation on blood pressure: a meta-analysis of randomized double-blind placebo-controlled trials. Hypertension 68:324-333

27. Taylor SI, Blau JE, Rother KI (2015) Possible adverse effects of SGLT2 inhibitors on bone. Lancet Diabetes Endocrinol 3:8-10

28. Bilezikian JP, Watts NB, Usiskin K et al (2016) Evaluation of bone mineral density and bone biomarkers in patients with type 2 diabetes treated with canagliflozin. J Clin Endocrinol Metab 101:44-51 